\title{
Kinematics of the faintest gas-rich galaxy in the Local Group: DD0210
}

\author{
A. Begum and J. N. Chengalur ${ }^{\star}$ \\ National Centre for Radio Astrophysics, Post Bag 3, Ganeshkhind, Pune 411 007, India \\ Received 16 July 2003 / Accepted 29 September 2003

\begin{abstract}
We present deep, high velocity resolution $\left(\sim 1.6 \mathrm{~km} \mathrm{~s}^{-1}\right)$ Giant Meterwave Radio Telescope (GMRT) HI $21 \mathrm{~cm}$ synthesis images of the faint $\left(M_{B} \sim-10.6\right)$ local group dwarf galaxy DDO210. We find that the HI distribution in the galaxy is not axi-symmetric, but shows density enhancements in the eastern and southern halves of the galaxy. The optical emission is lopsided with respect to the HI, most of the bright optical emission arises from the eastern half of the HI disk. The velocity field of the galaxy is however quite regular and shows a systematic large scale pattern, consistent with the rotational motion. The rotation curve for the galaxy shows a peak (inclination corrected) rotation velocity of only $\sim 8 \mathrm{~km} \mathrm{~s}^{-1}$, comparable to the random motions in the HI gas. After correcting for the dynamical support provided by random motions (the "asymmetric drift" correction), we find the corrected peak rotation velocity of $\sim 16.0 \mathrm{~km} \mathrm{~s}^{-1}$. Mass modeling of the corrected rotation curve shows that the kinematics of DDO210 can be well fit with either a modified isothermal halo (with a central density $\rho_{0} \sim$ $29 \times 10^{-3} M_{\odot} \mathrm{pc}^{-3}$ for a stellar mass to light ratio of 3.4) or an NFW halo. In the case of the NFW halo however, a good fit is obtained for a wide range of parameters; the halo parameters could not be uniquely determined from the fit. Density profiles with inner slope steeper than $\sim 1.2$ however provide a poor fit to the data. Finally, the rotation curve derived using MOND also provides a reasonable fit to the observed rotation curve.
\end{abstract}

Key words. galaxies: evolution - galaxies: dwarf - galaxies: kinematics and dynamics - galaxies: individual: DDO210 radio lines: galaxies

\section{Introduction}

Numerical simulations of hierarchical galaxy formation models such as the CDM model predict a "universal" cusped density core for the dark matter halos of galaxies (e.g. Navarro et al. 1996). A cusped density core corresponds to a steeply rising rotation curve. The observed kinematics of galaxies can hence be used to test such numerical models of galaxy formation. Dwarf low surface brightness (LSB), galaxies are best suited for such a test, since they, unlike larger galaxies, are known to be dark matter dominant. In large spiral galaxies, both gas and stars make significant contributions to the total mass, particularly in the inner regions of the galaxy. Since the exact contribution of the baryonic material to the total mass of the galaxy depends on the unknown mass to light ratio of the stellar disk, it is generally difficult to unambiguously determine the density profile of the dark matter halo in the central regions of large spirals. In dwarf LSB galaxies on the other hand, since the stellar disk is generally dynamically unimportant, the central halo density can be much better constrained. Interestingly, the observed rotation curves of dwarf galaxies generally indicate that their dark matter halos have constant density cores

Send offprint requests to: A. Begum,

e-mail: ayesha@ncra.tifr.res.in

* e-mail: chengalur@ncra.tifr.res.in (e.g. Weldrake et al. 2003) unlike the cusped density cores predicted in numerical simulations. Another prediction of the numerical simulations is that the density of the dark matter halo is related to the background density at the time of the halo formation. Since the smallest galaxies form first in such models, these galaxies are expected to have the largest halo densities. The determination of the shapes and characteristic densities of the dark matter halos of the faintest dwarf galaxies is hence a particularly interesting problem. However, a major stumbling block in such programs is that it is currently controversial whether very faint dwarf irregular galaxies show systematic rotation or not. Lo et al. (1993), in a study of the kinematics of a sample of nine faint dwarfs (with $M_{B} \sim-9.0$ to $M_{B} \sim-14.0$ ) found that most of them were characterized by chaotic velocity fields. However, as pointed out by Skillman (1996), Lo et al.'s observations had limited sensitivity to faint extended emission which is likely to have led to an underestimation of the rotation velocities. Further, from a recent high sensitivity and high velocity resolution study of the dwarf irregular galaxy, Camelopardalis B (Cam B), Begum et al. (2003) found that inspite of being very faint $\left(M_{B} \sim-10.8\right)$, the galaxy shows systematic rotation. Do all faint dwarf irregular galaxies have a rotating HI disk or is Cam B a special case? What is the dark matter distribution in these very faint galaxies? In this paper 
we discuss these questions in the specific context of the local group dwarf galaxy DDO210.

DDO210, the faintest known $\left(M_{B} \sim-10.6\right)$ gas rich dwarf galaxy in our local group, was discovered by van den Bergh (1959) and later detected in an HI $21 \mathrm{~cm}$ survey by Fisher \& Tully (1975). Fisher \& Tully (1979) assigned a distance of $0.7 \mathrm{Mpc}$ to it, based on its proximity to NGC 6822 both on the sky and in velocity. On the other hand, Greggio et al. (1993), based on the colour-magnitude (C-M) diagram of DDO210, estimated its distance to be $2.5 \mathrm{Mpc}$. However, recent distance estimates for DDO210 give distances closer to the original estimate of Fisher \& Tully (1979). Lee et al. (1999), based on the $I$ magnitude of the tip of the red giant branch, estimated the distance to DD210 to be $950 \pm 50 \mathrm{kpc}$. This estimate is in excellent agreement with the value of $940 \pm 40 \mathrm{kpc}$ derived recently by Karachentsev et al. (2002) using HST observations. At this distance, DDO210 would be a member of the local group.

DDO210 is classified as a dIr/dSph or "transition galaxy", with properties intermediate between dwarf irregulars and dwarf spheroidals (Mateo 1998). For example, in spite of containing a significant amount of neutral gas, DDO210 shows no signs of ongoing star formation. $\mathrm{H} \alpha$ imaging detected a single source of line emission in the galaxy; however follow up observations of this emission suggests that it does not arise in a normal HII region, but probably comes from dense outflowing material from an evolved star (van Zee et al. 1997). Consistent with the lack of ongoing starformation, the CM diagram of DDO210 shows that the brightest stars in the galaxy are the faintest among the brightest stars in all known dwarf irregular galaxies of our local group (Lee et al. 1999).

Prior to this work, there have been two HI interferometric studies of DDO210, both of which used the VLA. This galaxy was a part of the sample of Lo et al. (1993) discussed above, and has also been recently re-observed with a high velocity resolution in the Cs array (Young et al. 2003). Although Young et al. (2003) noted that DD210 showed a systematic large scale velocity gradient, no attempt was made to derive a rotation curve for the galaxy, since their study focused mainly on the local connection between the ISM and star formation.

We present here deep, high velocity resolution $\left(\sim 1.6 \mathrm{~km} \mathrm{~s}^{-1}\right)$ Giant Meterwave Radio Telescope (GMRT) observations of the HI emission from DDO210 and use them to study the kinematics of the neutral gas in this galaxy. The rest of the paper is divided as follows. The GMRT observations are detailed in Sect. 2, while the results are presented and discussed in Sect. 3. Throughout this paper we take the distance to DDO210 to be $1.0 \mathrm{Mpc}$, and hence its absolute magnitude to be $M_{B} \sim-10.6$.

\section{Observations}

The GMRT observations of DDO210 were conducted from 13-15 July 2002. The setup for the observations is given in Table 1. Absolute flux calibration was done using scans on the standard calibrators 3C 48 and 3C 286, one of which was observed at the start and end of each observing run. Phase
Table 1. Parameters of the GMRT observations.

\begin{tabular}{ll}
\hline \hline Parameters & Value \\
\hline RA(2000) & $20^{\mathrm{h}} 46^{\mathrm{m}} 53.0^{\mathrm{s}}$ \\
Declination(2000) & $-12^{\circ} 50^{\prime} 57^{\prime \prime}$ \\
Central velocity (heliocentric) & $-139.0 \mathrm{~km} \mathrm{~s}^{-1}$ \\
Date of observations & $13-15 \mathrm{July} 2002$ \\
Time on source & $16 \mathrm{hrs}$ \\
Total bandwidth & $1.0 \mathrm{MHz}$ \\
Number of channels & 128 \\
Channel separation & $1.65 \mathrm{~km} \mathrm{~s}{ }^{-1}$ \\
FWHM of synthesized beam & $44^{\prime \prime} \times 37^{\prime \prime}, 29^{\prime \prime} \times 23^{\prime \prime}$, \\
& $20^{\prime \prime} \times 15^{\prime \prime}, 12^{\prime \prime} \times 11^{\prime \prime}$ \\
RMS noise per channel & $2.2 \mathrm{mJy}, 1.8 \mathrm{mJy}, 1.6 \mathrm{mJy}$ \\
& $1.4 \mathrm{mJy}$ \\
\hline
\end{tabular}

calibration was done using the VLA calibrator source 2008-068 which was observed once every $30 \mathrm{~min}$. Bandpass calibration was done in the standard way using 3C 286.

The data were reduced using standard tasks in classic AIPS. For each run, bad visibility points were edited out, after which the data were calibrated. Calibrated data for all runs was combined using DBCON. The GMRT does not do online doppler tracking - any required doppler shifts have to be applied during the offline analysis. However since the differential doppler shift over our observing interval is much less than the channel width, there was no need to apply an offline correction.

The GMRT has a hybrid configuration (Swarup et al. 1991) with 14 of its 30 antennas located in a central compact array with size $\approx 1 \mathrm{~km}(\approx 5 \mathrm{k} \lambda$ at $21 \mathrm{~cm})$ and the remaining antennas distributed in a roughly " $\mathrm{Y}$ " shaped configuration, giving a maximum baseline length of $\approx 25 \mathrm{~km}(\approx 120 \mathrm{k} \lambda$ at $21 \mathrm{~cm})$. The baselines obtained from antennas in the central square are similar in length to those of the "D" array of the VLA while the baselines between the arm antennas are comparable in length to the "B" array of the VLA. A single observation with the GMRT hence yields information on both large and small angular scales. Data cubes were therefore made at various $(u, v)$ ranges, including $0-5 \mathrm{k} \lambda, 0-10 \mathrm{k} \lambda, 0-15 \mathrm{k} \lambda$ and $0-20 \mathrm{k} \lambda$ using uniform weighting. At each $(u, v)$ range, a circularly symmetric Gaussian taper with a $F W H M$ equal to $80 \%$ of the $(u, v)$ range was applied inorder to reduce the sidelobes of the synthesized beam. The angular resolutions obtained for the various $(u, v)$ ranges listed above were $44^{\prime \prime} \times 37^{\prime \prime}$, $29^{\prime \prime} \times 23^{\prime \prime}, 20^{\prime \prime} \times 15^{\prime \prime}$ and $12^{\prime \prime} \times 11^{\prime \prime}$ respectively. All the data cubes were deconvolved using the the AIPS task IMAGR. The $\mathrm{HI}$ emission from DDO210 spanned 22 channels of the spectral cube. A continuum image was made using the average of remaining line free channels. No continuum was detected from the disk of DDO210 to a $3 \sigma$ flux limit of $1.2 \mathrm{mJy} / \mathrm{Bm}$ (for a beam size of $\left.30^{\prime \prime} \times 23^{\prime \prime}\right)$. We also checked for the presence of any compact continuum sources in the disk of DDO210 by making a high resolution $\left(3.8^{\prime \prime} \times 3.0^{\prime \prime}\right)$ map - no sources 
associated with the disk of DDO210 were detected down to a $3 \sigma$ limit of $0.5 \mathrm{mJy} / \mathrm{Bm}$.

The line profiles were examined at various locations in the galaxy and were found (to zeroth order) to be symmetric and single peaked. Although double Gaussians or GaussHermite polynomials provide a better fit to the data (particularly in the higher column density regions), the peak velocity of the profile in these regions matches (within the errorbars) with the intensity weighted mean velocity. Since we are interested here mainly in the systematic velocities, moment maps provide an adequate description of the data. Moment maps were therefore made from the data cubes using the AIPS task MOMNT. To obtain the moment maps, lines of sight with a low signal to noise ratio were excluded by applying a cutoff at the $3 \sigma$ level, ( $\sigma$ being the rms noise level in a line free channel), after smoothing in velocity (using boxcar smoothing three channels wide) and position (using a Gaussian with $F W H M \sim 2$ times that of the synthesized beam). Maps of the velocity field and velocity dispersion were also made in GIPSY using single Gaussian fits to the individual profiles. The velocity field produced by Gaussian fitting is in reasonable agreement with that obtained from moment analysis. However, the moment 2 map systematically underestimates the velocity dispersion. This can be understood as the effect of the thresholding algorithm used by the MOMNT task to identify the regions with signal. The velocity dispersion from Gaussian fitting to the lines profiles was found to be $\sim 6.5 \mathrm{~km} \mathrm{~s}^{-1}$ and showed no measurable variation across the galaxy. This lack of substantial variation of $\sigma$ across DDO210 is typical of dwarf irregular galaxies, although the observed velocity dispersion for DDO210 is lower than the typical value observed in dwarf irregular galaxies (e.g. Lake et al. 1990; Skillman et al. 1988). A lower value of HI dispersion for DDO210 was also noted by Young et al. (2003).

\section{Results and discussion}

\subsection{HI distribution}

The global HI emission profile of DDO210, obtained from $44^{\prime \prime} \times 37^{\prime \prime}$ data cube, is shown in Fig. 1. A Gaussian fit to the profile gives a central velocity (heliocentric) of $-139.5 \pm$ $2.0 \mathrm{~km} \mathrm{~s}^{-1}$. The integrated flux is $12.1 \pm 1.2 \mathrm{Jy} \mathrm{km} \mathrm{s}^{-1}$. These are in good agreement with the values of $-140 \pm 2.0 \mathrm{~km} \mathrm{~s}^{-1}$ and $11.5 \pm 1.2 \mathrm{Jy} \mathrm{km} \mathrm{s}^{-1}$ obtained from single dish observations (Huchtmeier \& Richter 1986). A good agreement between the GMRT flux and the single dish flux shows that no flux was missed because of the missing short spacings in the interferometric observations. The velocity width at $50 \%$ level of peak emission $\left(\Delta V_{50}\right)$ is found to be $19.1 \pm 1 \mathrm{~km} \mathrm{~s}^{-1}$, which again is in excellent agreement with the $\Delta V_{50}$ value of $19.0 \mathrm{~km} \mathrm{~s}^{-1}$ from the single dish observations. The HI mass obtained from the integrated profile (taking the distance to the galaxy to be $1.0 \mathrm{Mpc}$ ) is $2.8 \pm 0.3 \times 10^{6} M_{\odot}$, and the $M_{\mathrm{HI}} / L_{\mathrm{B}}$ ratio is found to be $\sim 1.0$ in solar units.

Figure 2 shows the integrated HI emission from DDO210 at $44^{\prime \prime} \times 37^{\prime \prime}$ resolution, overlayed on the digitised sky survey (DSS) image. The HI isodensity contours are elongated

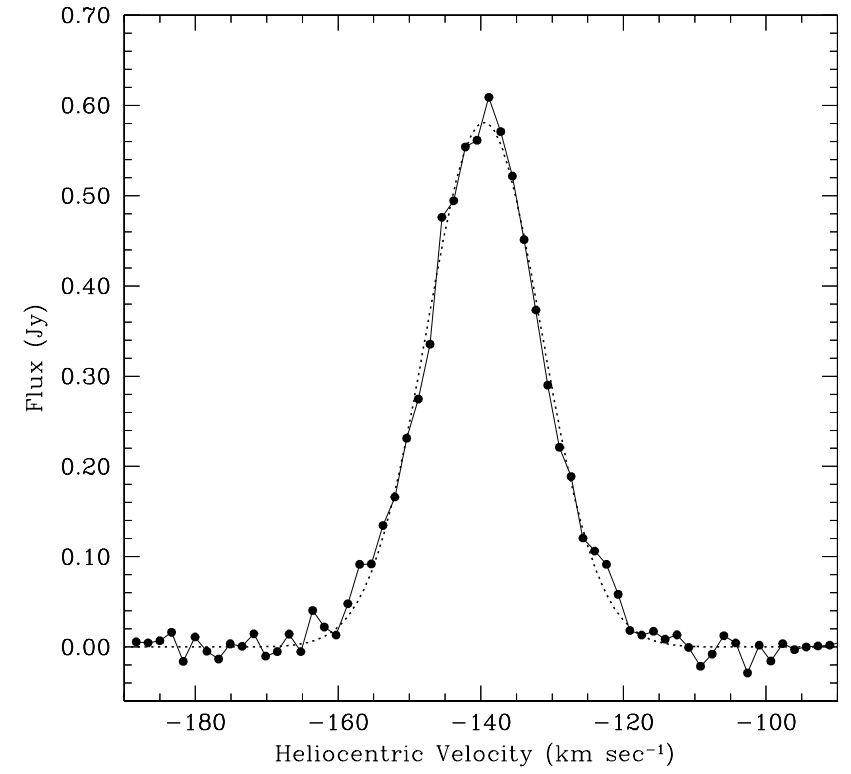

Fig. 1. HI profile for DDO 210 obtained from $44^{\prime \prime} \times 37^{\prime \prime}$ data cube. The channel separation is $1.65 \mathrm{~km} \mathrm{~s}^{-1}$. Integration of profile gives a flux integral of $12.1 \mathrm{Jy} \mathrm{km} \mathrm{s}^{-1}$ and an HI mass of $2.8 \times 10^{6} M_{\odot}$. The dashed line shows a Gaussian fit to the profile.

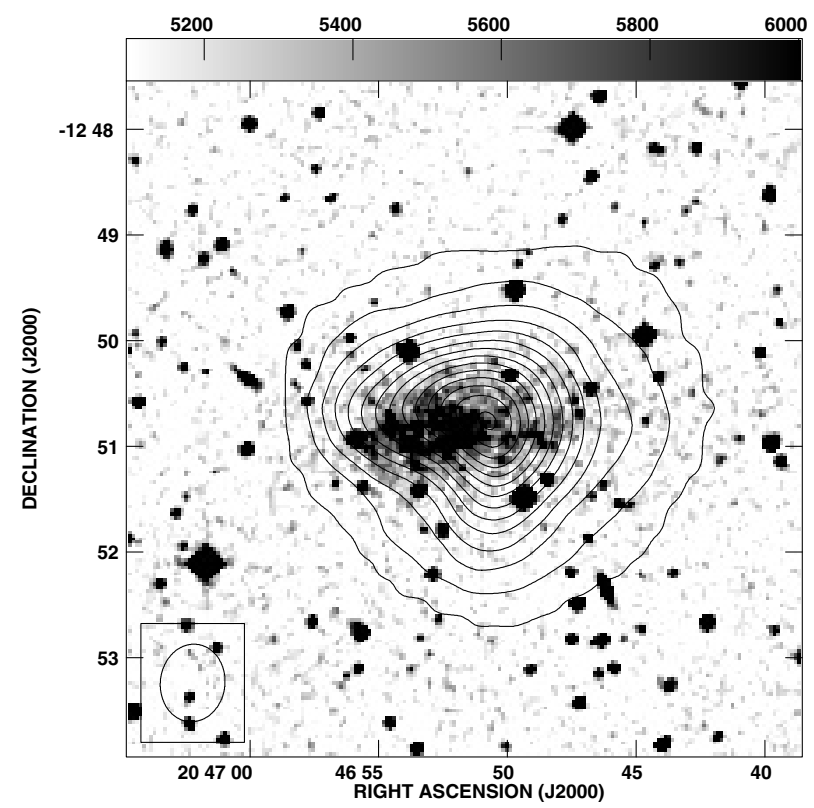

Fig. 2. The optical DSS image of DDO210 (greyscales) with the GMRT $44^{\prime \prime} \times 37^{\prime \prime}$ resolution integrated HI emission map (contours) overlayed. The contour levels are $0.01,0.151,0.293,0.434$, $0.576,0.717,0.854,0.999,1.141,1.283,1.424,1.565,1.797$, $1.848 \mathrm{Jy} / \mathrm{Bm} \mathrm{km} \mathrm{s}^{-1}$.

in eastern and southern half of the galaxy i.e. the density is enhanced in these directions. These density enhancements are highlighted in Fig. 4 which shows the integrated HI emission at high resolution $\left(12^{\prime \prime} \times 11^{\prime \prime}\right)$. Note that the faint extended emission seen in the low resolution map is resolved out in this image.

The optical emission in the galaxy shows two components, a central bright compact component and an outer faint 
extended component; both elongated in the east-west direction (Lee et al. 1999). However, the centers for the two components do not overlap; the bright component lies more eastwards than the center of extended component. From a deep C-M diagram of DDO210, Tolstoy et al. (2000) found a number of faint stars older than several Gyr in the galaxy. Van Zee (2000) estimated an inclination of 68 degrees for DDO210 from her observations. Lee et al. (1999), based on deeper observations, estimated the inclination of the faint extended component to be $\sim 60$ degrees, but noted that the presence of several bright foreground stars makes this estimate of inclination very uncertain.

From a visual inspection of the overlay (Fig. 2), the eastern HI density enhancements seem to correlate with the optical emission in the galaxy. As can be seen the bright component of the optical emission is not centered on the HI emission, but is instead offset to the east. On the other hand, no optical emission is seen along the southern HI density enhancement. As a further check for optical emission associated with the southern HI density enhancement, we computed number counts of stars in that region using the deep optical image obtained by Tolstoy et al. (2000). No significant increase in the star counts from the background was detected.

As seen in Fig. 2, the faint extended emission in the lower resolution HI distribution is less distorted by the presence of the HI density enhancements. Hence, an estimate of the morphological center, position angle (PA) and inclination of the galaxy was obtained by fitting elliptical annuli to the $44^{\prime \prime} \times 37^{\prime \prime}$ resolution HI distribution. The estimated HI morphological center lies close to the center of the faint extended optical component (but as noted above is offset from the center of the bright optical emission). The variation of the fitted morphological PA with the galactocentric radius is given in Fig. 5. The inclination estimated from the outer HI contours (assuming the intrinsic shape of the HI disk to be circular) is $27 \pm 7.0$ degrees. The inclination in the inner regions of the galaxy could not be constrained reliably due to the significant distortion in the HI contours. For the same reason, ellipse fitting to the higher resolution $\mathrm{HI}$ data (where the smooth emission is resolved out) is not reliable.

The inclination derived from the HI distribution is significantly different from the optical inclination of the galaxy. It is likely that the optical emission does not arise from an axisymmetric disk, but instead that the stars are concentrated in a patchy elongated region in DDO210. Hence, in the absence of any other reliable estimate, the value of inclination obtained from the outer HI contours was assigned to the whole galaxy. The deprojected HI radial surface density profiles were then obtained by averaging over elliptical annuli in the plane of the galaxy. The profiles derived from the $44^{\prime \prime} \times 37^{\prime \prime}$ and $29^{\prime \prime} \times 23^{\prime \prime}$ resolution $\mathrm{HI}$ distributions are given in Fig. 3. As can be seen, the two distributions are in reasonable agreement. The flux integral estimated from the $29^{\prime \prime} \times 23^{\prime \prime}$ resolution HI moment 0 map was found to be $\sim 11 \%$ smaller than that estimated from the $44^{\prime \prime} \times 37^{\prime \prime}$ resolution HI distribution. For the rest of the analysis, we will use the $44^{\prime \prime} \times 37^{\prime \prime} \mathrm{HI}$ profile. This profile is well represented by a Gaussian, i.e. we have

$\Sigma_{\mathrm{HI}}(r)=\Sigma_{0} \times \mathrm{e}^{-(r-c)^{2} / 2 r_{0}^{2}}$

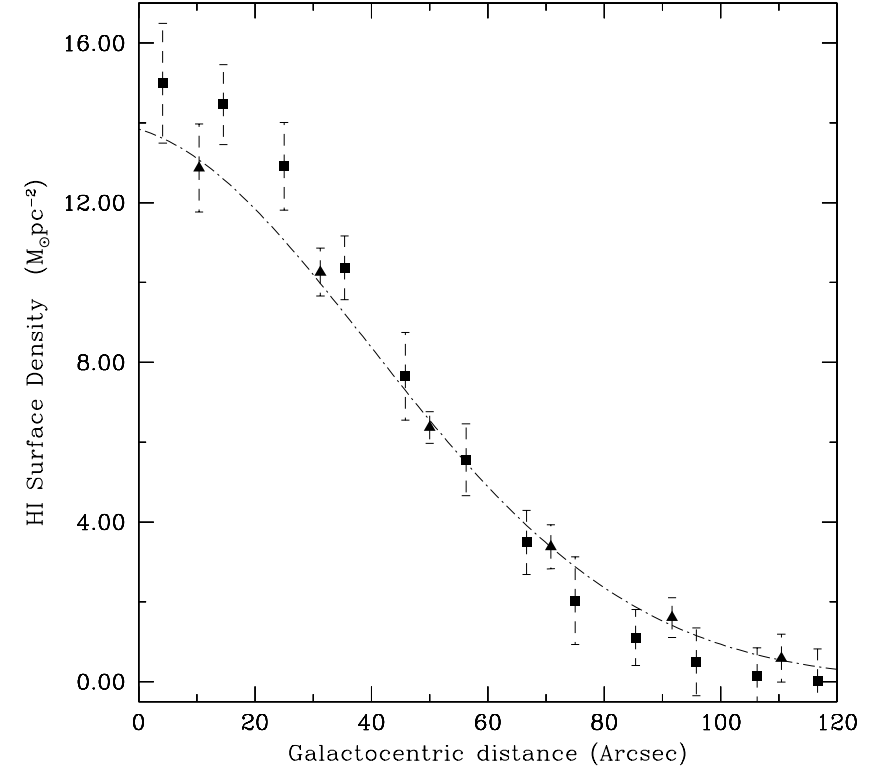

Fig. 3. The HI surface density profile derived from the HI distribution at $29^{\prime \prime} \times 23^{\prime \prime}$ (squares) and $44^{\prime \prime} \times 37^{\prime \prime}$ (triangles) resolution. A Gaussian fit to $44^{\prime \prime} \times 37^{\prime \prime} \mathrm{HI}$ distribution is shown superimposed.

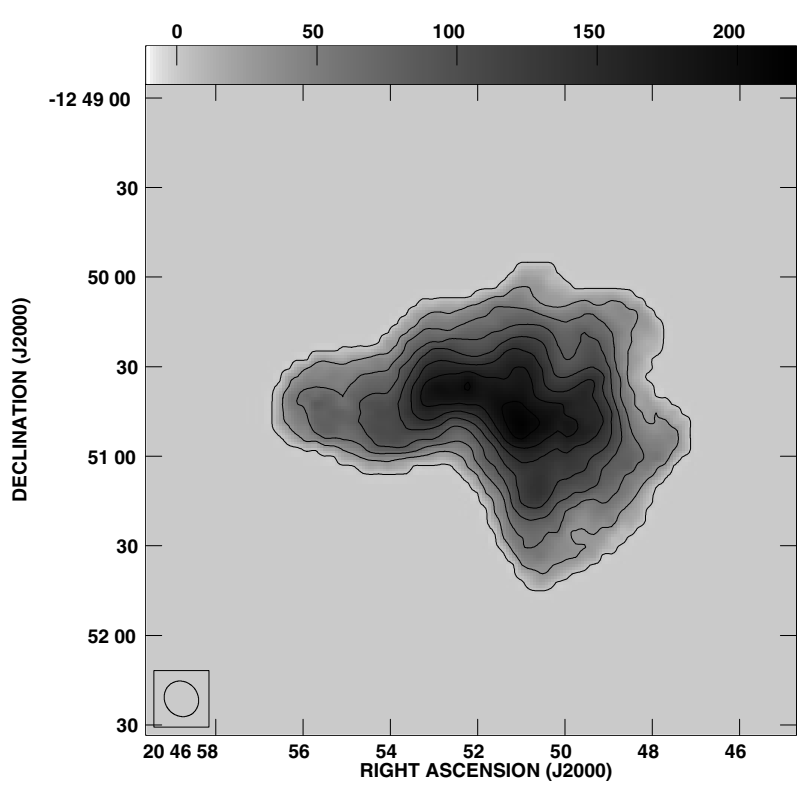

Fig. 4. Integrated HI emission map of DDO210 (grey scale and contours) at $12^{\prime \prime} \times 11^{\prime \prime}$ resolution. The contour levels are $0.001,0.154$, $0.293,0.434,0.576,0.717,0.858,0.999,1.141 \mathrm{Jy} / \mathrm{Bm} \mathrm{km} \mathrm{s}^{-1}$.

with $\Sigma_{0}=13.9 \pm 1.0 M_{\odot} \mathrm{pc}^{-2}, c=-4.0^{\prime \prime}(\sim 0.02 \mathrm{kpc})$ and $r_{0}=46^{\prime \prime}(\sim 0.22 \mathrm{kpc})$.

\subsection{HI kinematics}

The velocity field of DDO210 derived from the moment analysis of $29^{\prime \prime} \times 23^{\prime \prime}$ resolution data cube is shown in Fig. 6 . The velocity field is regular and a systematic velocity gradient is seen across the galaxy. Our velocity field differs significantly from the velocity field derived by Lo et al. (1993). The systematic pattern seen in our velocity field is, to zeroth order, consistent with that expected from rotation. On the other hand, the 


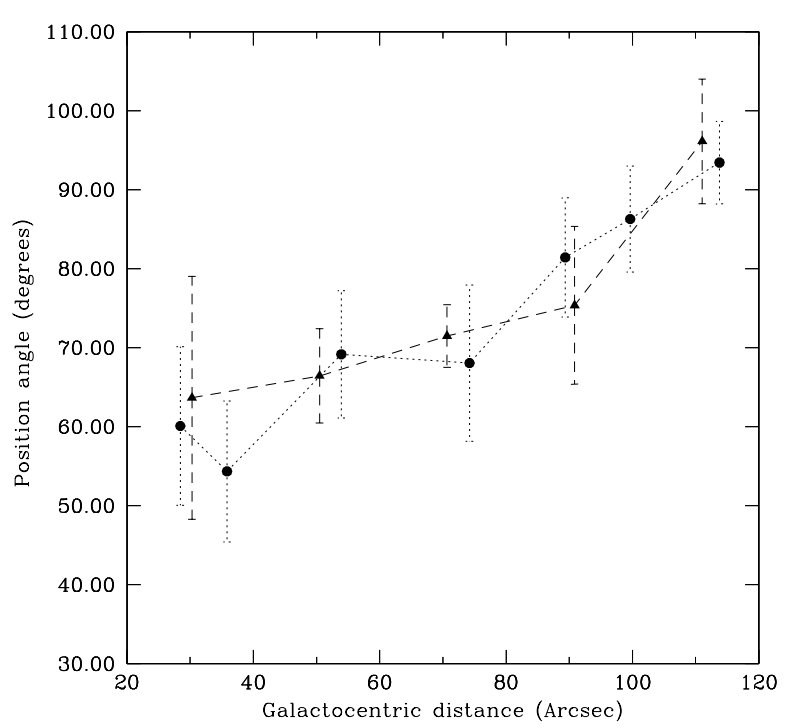

Fig. 5. The variation of position angle (PA) with the galactocentric distance. Circles represent the morphological PA obtained from ellipse fit to the HI distribution at $44^{\prime \prime} \times 37^{\prime \prime}$ resolution. Triangles represent the kinematical PA derived from $44^{\prime \prime} \times 37^{\prime \prime}$ resolution velocity field.

velocity field derived by Lo et al. (1993) (based on a coarser velocity resolution of $\sim 6 \mathrm{~km} \mathrm{~s}^{-1}$ ) is chaotic. This difference in the observed kinematics suggests that high velocity resolution and high sensitivity is crucial in determining the systematic gradients in the velocity field of faint galaxies like DDO210. A similar systematic velocity pattern was also found in the recent high velocity resolution study of DDO210 by Young et al. (2003), however, those authors concentrated on trying to determine the physical conditions of the emitting gas and not the large scale kinematics of the galaxy.

The velocity field in Fig. 6 also shows signatures of both warping and kinematical lopsidedness (Swaters et al. 1999), i.e. the kinematical major axis is not a straight line but shows twists and the isovelocity contours in the eastern half of the galaxy are more closed than those in the western half. Both of these kinematical peculiarities become more prominent in the higher spatial resolution velocity field (see Fig. 8). While the density enhancements in the $\mathrm{HI}$ also become more prominent at higher spatial resolutions, there does not seem to be any particular correlation between the HI density enhancements and these kinematical peculiarities.

\subsection{HI Rotation curve}

Given the lack of correlation between the density enhancements in the HI distribution and the global kinematical peculiarities in DDO210, it would be reasonable to assume that these density enhancements follow the same kinematics as that of the more diffuse extended emission. Under the further assumption that the kinematical peculiarities noted above are not important (to the zeroth order; see also Sect. 3.5), rotation curves were derived by fitting the usual tilted ring model to the HI velocity fields at various resolutions (including $44^{\prime \prime} \times 37^{\prime \prime}$, $29^{\prime \prime} \times 23^{\prime \prime}, 20^{\prime \prime} \times 15^{\prime \prime}$ and $\left.12^{\prime \prime} \times 11^{\prime \prime}\right)$ using the GIPSY task ROTCUR.

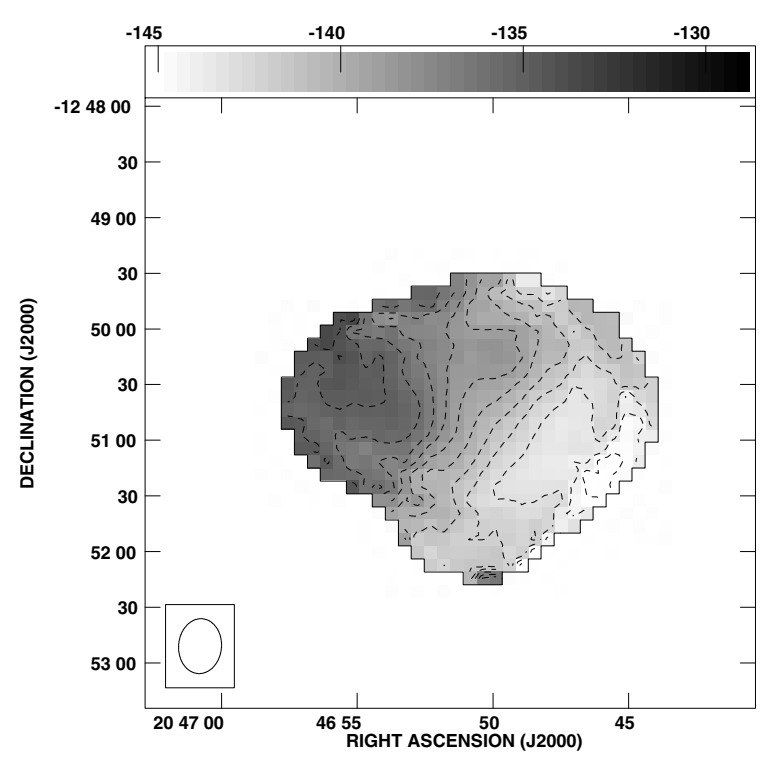

Fig. 6. The HI velocity field of DDO 210 at $29^{\prime \prime} \times 23^{\prime \prime}$ resolution. The contours are in steps of $1 \mathrm{~km} \mathrm{~s}^{-1}$ and range from $-145.0 \mathrm{~km} \mathrm{~s}^{-1}$ to $-133.0 \mathrm{~km} \mathrm{~s}^{-1}$.

First, the kinematical center and the systemic velocity $\left(V_{\text {sys }}\right)$ of DDO210 were obtained from a global fit to the velocity fields at various resolutions. The values of $V_{\text {sys }}$ derived from the velocity fields matched within the errorbars and also matched with the value obtained from a Gaussian fit to the global HI profile. The kinematical center derived from a global fit to the velocity fields at $44^{\prime \prime} \times 37^{\prime \prime}$ and $29^{\prime \prime} \times 23^{\prime \prime}$ resolution matched within the errorbars and also matched with the morphological center derived from the $44^{\prime \prime} \times 37^{\prime \prime}$ resolution HI distribution. However, because of distorted morphology of DDO210 at high spatial resolutions, an attempt to derive the kinematical center from a global fit to the velocity fields at these resolutions did not yield reliable results. Hence, the center for the higher resolution velocity fields was fixed to a value obtained from the lower resolution velocity fields.

Keeping $V_{\text {sys }}$ and the kinematical center fixed to the values obtained from the global fit, the position angle (PA) of the galaxy was derived, using tilted ring model, by breaking up the galaxy into elliptical annuli (each of width half that of the synthesized beam). The variation of the derived kinematical PA with the galactocentric radius for the $44^{\prime \prime} \times 37^{\prime \prime}$ resolution velocity field is given in the Fig. 5. The value of kinematical PA at all galactocentric radii matched with the morphological PA estimated from the $44^{\prime \prime} \times 37^{\prime \prime}$ resolution HI distribution. Attempts to derive the kinematical position angle at the higher spatial resolutions did not yield reliable estimates. Similarly, attempts to derive the kinematical inclination of DDO210 did not give reliable results at any spatial resolution. Hence, in the absence of any other reliable estimate, the kinematical inclination of the HI disk was fixed to the value obtained from the HI morphology. Finally, the rotation curve of the galaxy was computed keeping all parameters, except the circular velocity $\mathrm{V}_{c}$, in each elliptical annuli fixed.

Figure 7 shows the rotation curves derived from the different spatial resolution velocity fields. As can be seen, 


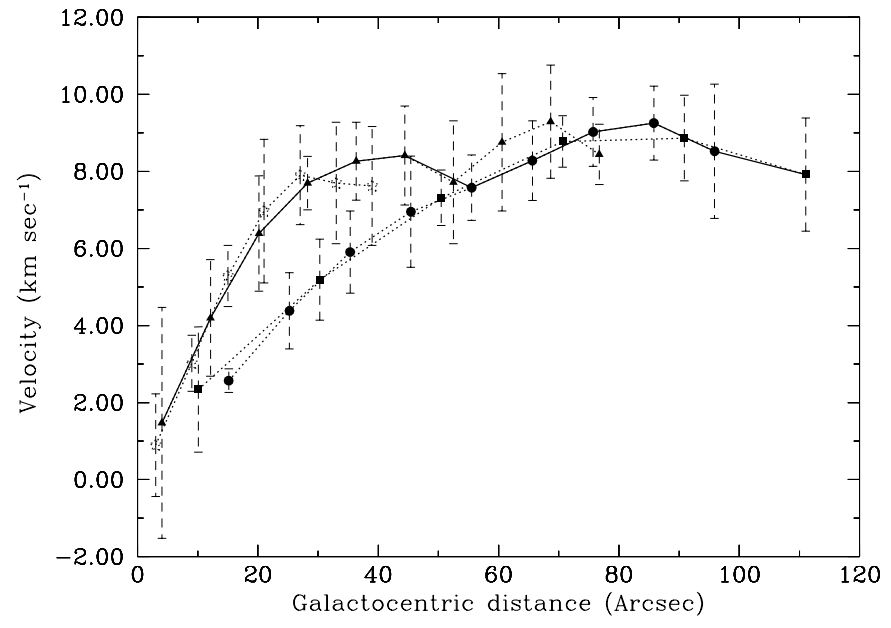

Fig. 7. The rotation curves derived from the intensity weighted velocity field at various resolutions. Crosses, triangles, circles and squares show the rotation velocity derived from the $12^{\prime \prime} \times 11^{\prime \prime}, 20^{\prime \prime} \times 15^{\prime \prime}$, $29^{\prime \prime} \times 23^{\prime \prime}$ and $44^{\prime \prime} \times 37^{\prime \prime}$ resolution respectively. The adopted hybrid rotation curve is shown by a solid line.

the $20^{\prime \prime} \times 15^{\prime \prime}$ and $12^{\prime \prime} \times 11^{\prime \prime}$ resolution velocity fields lead to the rotation curves that are significantly steeper in the inner regions of the galaxy than those computed from the lower resolution $44^{\prime \prime} \times 37^{\prime \prime}$ and $29^{\prime \prime} \times 23^{\prime \prime}$ velocity fields; this could be a result of beam smearing. On the other hand, the rotation curves from each resolution agree in the outer regions of the galaxy. This suggests that the effect of beam smearing is significant only in the inner regions of the galaxy, where the rotation velocity is increasing with the galactocentric radius. Further, the rotation curves derived from the $12^{\prime \prime} \times 11^{\prime \prime}$ resolution velocity field agrees with that obtained from the $20^{\prime \prime} \times 15^{\prime \prime}$ resolution data suggesting that beam smearing effects are no longer significant at such high resolutions (which corresponding to a linear scale of $\sim 70 \mathrm{pc}$ ). The final rotation curve that we adopt for the rest of the analysis is shown as a solid line in Fig. 7. The rotation velocities derived from the $20^{\prime \prime} \times 15^{\prime \prime}$ resolution velocity field are used in the inner regions of the galaxy (upto $\sim 50^{\prime \prime}$ ), while, the outer points are taken from the $29^{\prime \prime} \times 23^{\prime \prime}$ and the $44^{\prime \prime} \times 37^{\prime \prime}$ resolution data.

As discussed above, the rotation curves have been derived under the assumption that the HI density enhancements follow the same kinematics as that of the extended emission. The validity of this assumption could be tested by checking whether the derived rotation curves can reproduce the observed kinematics in the galaxy. Hence, model velocity fields were made at $44^{\prime \prime} \times 37^{\prime \prime}, 29^{\prime \prime} \times 23^{\prime \prime}$ and $20^{\prime \prime} \times 15^{\prime \prime}$ resolutions using the rotation curves and other kinematical parameters, derived from each resolution with a tilted ring model, using the task VELFI in GIPSY. The model velocity field at $20^{\prime \prime} \times 15^{\prime \prime}$ resolution is shown in Fig. 8. As can be seen from the figure, the model provides a reasonable match to the observed velocity field. Note however that the model fails to reproduce the kinematical lopsidedness and the twist in the kinematical major axis. This is to be expected, since neither of these two features was incorporated in the model. A similar match between the observed and the model velocity fields was also found for the two lower resolution velocity fields.

The maximum rotation velocity (inclination corrected) of DDO210 is $\sim 8.0 \mathrm{~km} \mathrm{~s}^{-1}$, which is comparable to the velocity dispersion observed in the $\mathrm{HI}$ gas, i.e. random motion in the gas provides significant dynamical support to the HI disk. Since the pressure support contributes significantly to the dynamics of DDO210, the observed rotation velocities underestimate the total dynamical mass. The rotation velocity hence has to be corrected for the pressure support before one can derive a mass model for the galaxy; this correction (generally called the "asymmetric drift" correction) is given by:

$v_{\mathrm{c}}^{2}=v_{\mathrm{o}}^{2}-r \times \sigma^{2}\left[\frac{\mathrm{d}}{\mathrm{d} r}\left(\ln \Sigma_{\mathrm{HI}}\right)+\frac{\mathrm{d}}{\mathrm{d} r}\left(\ln \sigma^{2}\right)-\frac{\mathrm{d}}{\mathrm{d} r}\left(\ln 2 h_{z}\right)\right]$,

where $v_{\mathrm{c}}$ is the corrected circular velocity, $v_{\mathrm{o}}$ is the observed rotation velocity, $\sigma$ is the velocity dispersion, and $h_{z}$ is the scale height of the disk. Strictly speaking, asymmetric drift corrections are applicable to collisionless stellar systems for which the magnitude of the random motions is much smaller than that of the rotation velocity. However, it is often used even for gaseous disks, where the assumption being made is that the pressure support can be approximated as the gas density times the square of the random velocity. In the absence of any measurement for $h_{z}$ for DDO210, we have assumed $\mathrm{d}\left(\ln \left(h_{z}\right)\right) / \mathrm{d} r=0$ (i.e. that the scale height does not change with radius). Also, using the fact that $\sigma$ is constant across the galaxy, we get:

$v_{\mathrm{c}}^{2}=v_{\mathrm{o}}^{2}-r \times \sigma^{2}\left[\frac{\mathrm{d}}{\mathrm{d} r}\left(\ln \Sigma_{\mathrm{HI}}\right)\right]$.

Using the fitted Gaussian profile to the radial surface density distribution, (see Eq. (1)) we obtain

$v_{\mathrm{c}}^{2}=v_{\mathrm{o}}^{2}+r(r-c) \times \sigma^{2} / r_{0}^{2}$.

The observed HI velocity dispersion was corrected for the instrumental broadening as well as for the broadening due to the velocity gradient over the finite size of the beam. The applied correction is $\sigma_{\text {true }}^{2}=\sigma_{\text {obs }}^{2}-\Delta v^{2}-\frac{1}{2} b^{2}\left(\nabla v_{0}\right)^{2}$, where $\sigma_{\text {true }}$ is the true velocity dispersion, $\Delta v$ is the channel width, $b$ characterizes the beam width (i.e. the beam is assumed to be of form $\mathrm{e}^{-x^{2} / b^{2}}$ ) and $v_{\mathrm{o}}$ is the observed rotation velocity. After putting the appropriate values in the above equation, we get $\sigma_{\text {true }}^{2} \approx 36.0 \mathrm{~km}^{2} \mathrm{~s}^{-2}$. Finally, substituting this value back into the Eq. (4) the "asymmetric drift" corrected curve obtained is given as a dotted line in Fig. 9.

As discussed above, the "asymmetric drift" correction was derived under the assumption that the disk scale height of DDO210 does not change with radius. To quantify the effect of this assumption, the correction was recalculated assuming a linear increase of $100 \%$ in the scale height, from $q_{0}=0.25$ at the center to $q_{0}=0.5$ at the edge of the galaxy. The change in the asymmetric drift correction was found to be $<1 \%$. The assumption that the scale height is constant hence has a negligible effect on the derived halo parameters. 

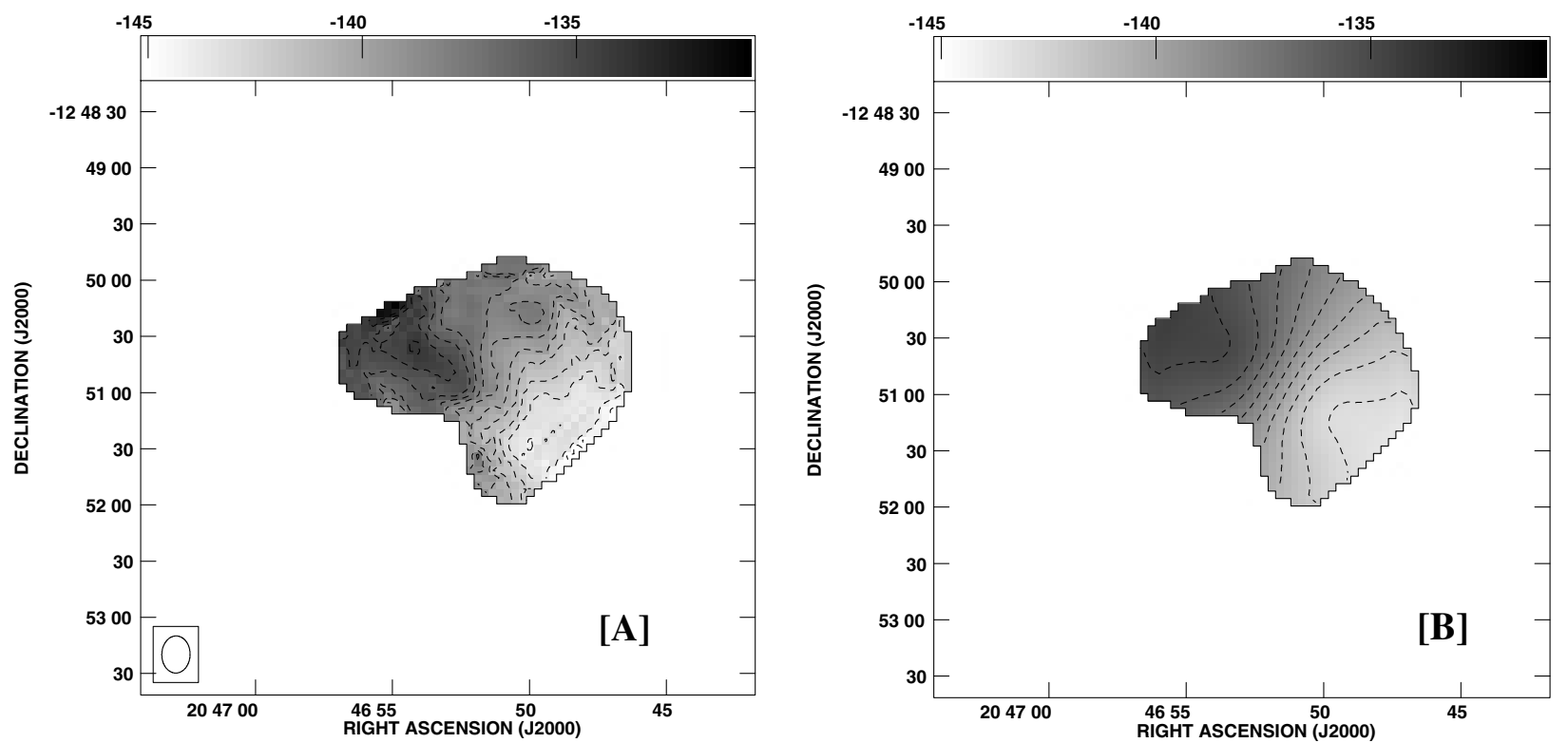

Fig. 8. [A] The observed velocity field of DDO210 at $20^{\prime \prime} \times 15^{\prime \prime}$ arcsec resolution. [B] The model velocity field derived from the rotation curve at $20^{\prime \prime} \times 15^{\prime \prime}$ resolution. The contours are in steps of $1 \mathrm{~km} \mathrm{~s}^{-1}$ and range from $-144.0 \mathrm{~km} \mathrm{~s}^{-1}$ to $-134.0 \mathrm{~km} \mathrm{~s}^{-1}$.

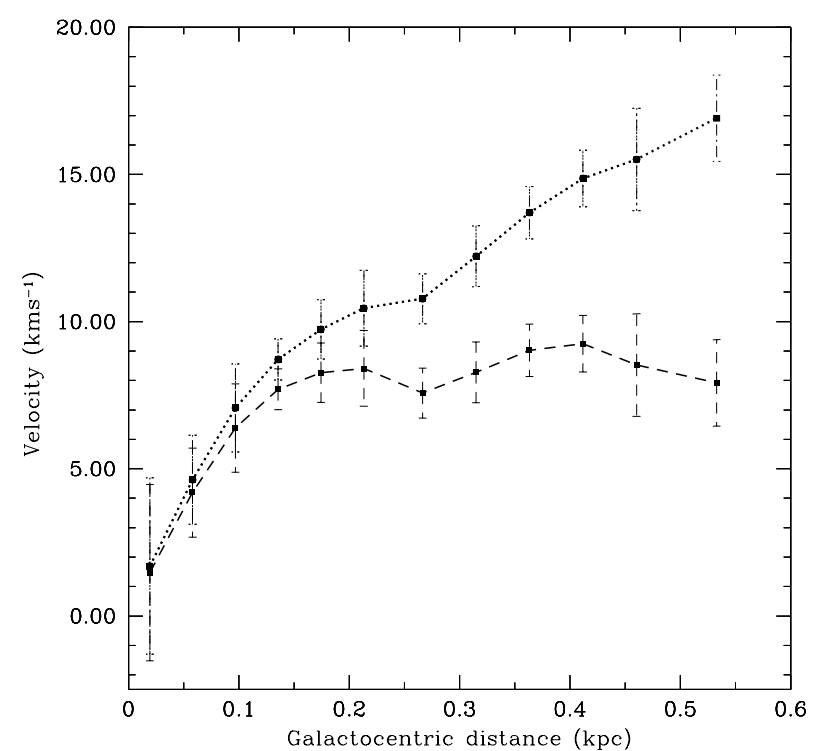

Fig. 9. The hybrid rotation curve (dashes) and the rotation curve after applying the asymmetric drift correction (dots).

\subsection{Mass model}

In this section we use the "asymmetric drift" corrected rotation curve derived in the last section to derive mass models for DDO210.

As discussed in Sect. 3.1 the optical emission is not axi-symmetric but is instead patchy and elongated. For the purpose of mass modeling however, we approximate the optical emission to be axi-symmetric, but we return to this issue in Sect. 3.5. Assuming that the optical emission was axi-symmetric, Lee et al. (1999) estimate the $B$ band scale length $\left(\alpha_{B}\right)$ of DDO210 to be $35.0^{\prime \prime}(\sim 0.17 \mathrm{kpc})$. This estimate of $\alpha_{B}$ agrees to within $10 \%$ with the value of $39^{\prime \prime}$ estimated by Van Zee (2000). Lee et al. (1999) also found the colours of the galaxy to be nearly constant with the galactocentric radius. Hence, we computed the contribution of the stellar mass to the observed rotation curve by assuming it to be an exponential disk of constant $\mathrm{M} / \mathrm{L}_{B}$ ratio $\left(\Upsilon_{B}\right)$ and with an intrinsic thickness ratio $\left(q_{0}\right)$ of 0.25 . For the vertical density distribution of the stellar disk, we assumed $\operatorname{sech}^{2}\left(z / z_{0}\right)$ profile, with $z_{0}$ independent of galactocentric radius. These are reasonable assumptions for disk galaxies (e.g. van der Kruit \& Searle 1981; de Grijs \& Peletier 1997). In the absence of any prior knowledge of $\Upsilon_{B}$, it was taken as a free parameter in the mass modelling.

The contribution of the HI mass to the observed rotation velocities was calculated using the HI surface density profile derived from $44^{\prime \prime} \times 37^{\prime \prime} \mathrm{HI}$ distribution. To correct for the mass fraction of helium, the HI mass was scaled by a factor of 1.25. A search for molecular gas in DDO210 gave negative result (Taylor et al. 1998), hence, the contribution of molecular gas to the rotation curve was ignored. We also neglected the contribution of ionized gas, if any. Not much is known about the vertical distribution of gas in dwarf irregular galaxies, however there is some evidence of similar vertical density distribution of $\mathrm{HI}$ and stellar disk (Bottema et al. 1986). Hence, for the HI disk we again assumed $q_{0}$ of 0.25 , with a vertical density distribution profile of $\operatorname{sech}^{2}\left(z / z_{0}\right)$. The rotation velocities for the HI and the stellar components were then computed using the formulae given by Casertano (1983).

For the dark matter halo we considered two types of density profiles, viz. the modified isothermal profile and the NFW profile. The modified isothermal density profile is given by:

$\rho_{\text {iso }}(r)=\rho_{0}\left[1+\left(r / r_{\mathrm{c}}\right)^{2}\right]^{-1}$,

where, $\rho_{0}$ is the central density of the halo and $r_{\mathrm{c}}$ is the core radius. The corresponding circular velocity is given by:

$v(r)=\sqrt{4 \pi G \rho_{0} r_{\mathrm{c}}^{2}\left[1-\frac{r}{r_{\mathrm{c}}} \tan ^{-1}\left(\frac{r}{r_{\mathrm{c}}}\right)\right]}$. 


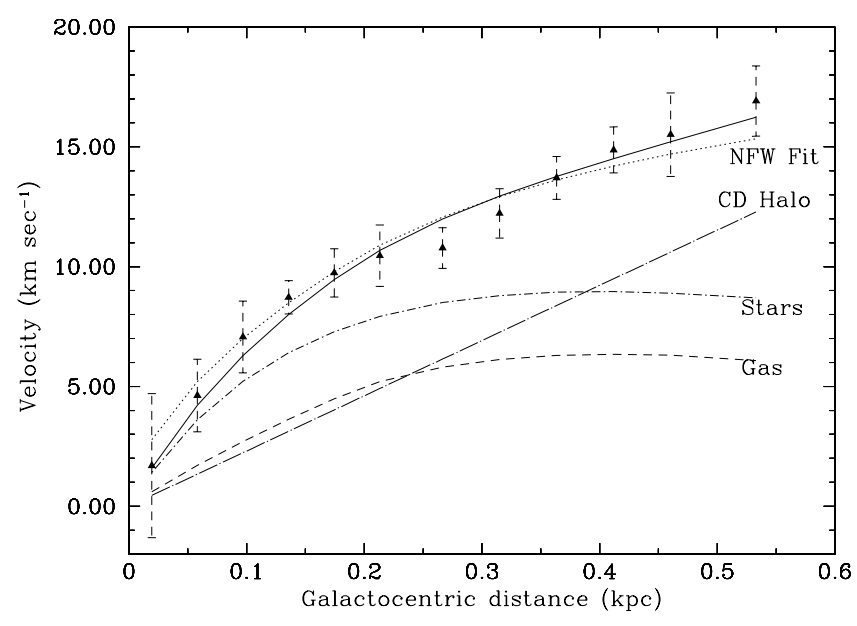

Fig. 10. Mass models for DDO210 using the corrected rotation curve. The points are the observed data. The total mass of gaseous disk (dashed line) is $3.6 \times 10^{6} M_{\odot}$. The stellar disk (short dash dot line) has $\Upsilon_{B}=3.4$, giving a stellar mass of $9.2 \times 10^{6} M_{\odot}$. The best fit total rotation curve for the constant density halo model is shown as a solid line, while the contribution of the halo itself is shown as a long dash dot line (the halo density is density $\rho_{0}=29 \times 10^{-3} M_{\odot} \mathrm{pc}^{-3}$ ). The best fit total rotation curve for an NFW type halo, using $\Upsilon_{B}=0.5, c=5.0$ and $v_{200}=38.0 \mathrm{~km} \mathrm{~s}^{-1}$ is shown as a dotted line. See the text for more details.

The NFW halo density profile is given by:

$\rho_{\mathrm{NFW}}(r)=\rho_{i} /\left[\left(r / r_{s}\right)\left(1+r / r_{s}\right)^{2}\right]$,

where, $r_{s}$ is the characteristic radius of the halo and $\rho_{i}$ is the characteristic density. The circular velocity can be written as:

$v(x)=v_{200} \sqrt{\frac{\ln (1+c x)-c x /(1+c x)}{x[\ln (1+c)-c /(1+c)]}}$,

where, $c=r_{200} / r_{s}, x=r / r_{200} ; r_{200}$ is the distance at which the mean density of the halo is equal to 200 times the critical density and $v_{200}$ is the circular velocity at this radius.

First, we consider a modified isothermal dark halo mass model. Since the rotation curve keeps on rising till the last measured point (see Fig. 9), the core radius $r_{\mathrm{c}}$ could not be constrained. This essentially reduces a modified isothermal dark halo fit into a constant density halo fit. The remaining free parameters are the central density of the halo, $\rho_{0}$, and the mass to light ratio of the stellar disk, $\Upsilon_{B}$.

Figure 10 shows the best fit mass model for a constant density halo. The best fit gave $\Upsilon_{B}$ of $3.4 \pm 0.5$ and $\rho_{0}=$ $29 \pm 5 \times 10^{-3} M_{\odot} \mathrm{pc}^{-3}$. The observed B-V for DDO210 is $\sim 0.25$ (Lee et al. 1999), which, (from the low metallicity Bruzual \& Charlot SPS model using a modified Salpeter IMF, Bell $\&$ de Jong 2001), corresponds to a $\Upsilon_{B}$ of $\sim 0.5$. If we adopt this value of $\Upsilon_{B}$ then the best fit model has $\rho_{0}=59 \pm 7 \times$ $10^{-3} M_{\odot} \mathrm{pc}^{-3}$. However, with this $\Upsilon_{B}$, the fit to the observed rotation curve is much poorer and the model curve substantially underestimates the observed rotation velocities at small radii. The best fit model $\left(\Upsilon_{B}=3.4\right)$ gives the mass of stellar disk to be $M_{*}=9.2 \times 10^{6} M_{\odot}$. The mass of the gas disk in DDO210 is $M_{\text {gas }}=3.5 \times 10^{6} M_{\odot}$. From the last measured point of the observed rotation curve, we get a total dynamical mass of $M_{\mathrm{T}}=3.4 \times 10^{7} M_{\odot}$, i.e. at the last measured point more than $63 \%$ of the mass of DDO210 is dark.

A similar procedure was also tried using a dark matter halo of the NFW type. Keeping $\Upsilon_{B}$ as a free parameter in the fit gave unphysical results, hence, it was kept fixed to a more likely value (viz. $\Upsilon_{B}=0.5$ ). We found that an NFW halo provides a good fit to the data, for a wide range of values of $v_{200}$ and $c$. The range of parameters which provide acceptable fits are $\left(v_{200} \sim 20 \mathrm{~km} \mathrm{~s}^{-1}, c \sim 10\right)$ to $\left(v_{200} \sim 500 \mathrm{~km} \mathrm{~s}^{-1}, c \sim 0.001\right)$. As an illustration we show in Fig. 10 the best fit rotation curve for an NFW halo, using $\Upsilon_{B}=0.5, c=5, v_{200}=38 \mathrm{~km} \mathrm{~s}^{-1}$. As can be seen clearly, the NFW halo also provides a good fit to the data. However, the best fit values of the concentration parameter $\mathrm{c}$, at any given $v_{200}$ was found to be consistently smaller than the value predicted by numerical simulations for the $\Lambda$ CDM universe (Marchesini et al. 2002).

As seen above, both isothermal and NFW halo provide a good fit to the observed kinematics of DDO210. Profiles steeper than NFW have also been proposed by some N-body simulations (e.g. Moore et al. 1999). In order to check whether such steep profiles are also consistent with the data, mass models were fit using a broader family of density profiles, viz.

$\rho(r)=\frac{\rho_{0}}{\left(r / r_{0}\right)^{\alpha}\left[1+\left(r / r_{0}\right)^{\gamma}\right]^{(\beta-\alpha) / \gamma}}$.

The circular velocity corresponding to the above density profile (see Kravtsov et al. 1998) is:

$V(r)=V_{\mathrm{t}} \frac{\left(r / r_{\mathrm{t}}\right)^{g}}{\left[1+\left(r / r_{\mathrm{t}}\right)^{a}\right]^{(g+b) / a}}$

where $r_{\mathrm{t}}$ and $V_{\mathrm{t}}$ are the effective "turnover" radius and velocity. The parameter " $g$ " is related to the inner slope of the density profile, $\alpha$ by $g=1+\alpha / 2.0$, " $b$ " is the outer logarithmic slope of the rotation curve while " $a$ " determines the sharpness of turnover. Fits to the rotation curve with all three parameters left free did not converge. Hence, since we are primarily concerned with the slope in the inner regions, we fixed the parameters $b$ and a to the values of 0.34 and 1.5 respectively, (which are the typical values found for the rotation curves of dwarf galaxies; Kravtsov et al. 1998), while $r_{\mathrm{t}}$ and $V_{\mathrm{t}}$ were left as free parameters. $\Upsilon_{B}$ was fixed to a value of 0.5 , as suggested by the observed colours in the galaxy; this also allows a meaningful comparison of derived mass models for a family of density profiles. We found that the reduced $\chi^{2}$ for the fit continuously increases as the profile gets steeper. For comparison, fixing $g$ to 0.5 (corresponding to $\alpha=1.0$; NFW profile) gave reduced $\chi^{2}=0.4$ while $\mathrm{g}$ of 0.4 (corresponding to $\alpha=1.2$ ) gave reduced $\chi^{2}=0.7$. At the extreme, fixing g to $0.2(\alpha=1.6)$ (which substantially over-predicts the observed rotation velocity at small radii, while underestimates the velocity at large radii) gave reduced $\chi^{2}=2.5$. Note that since there are heuristics involved in computing the error bars on $v_{\mathrm{c}}$, it is not possible to rigorously translate the minimum $\chi^{2}$ value into a confidence interval for the parameters of the fit. However, a lower $\chi^{2}$ value does imply a better fit (see also the discussion in van den Bosh $\&$ Swaters 2001). Figure 11 shows the best fit mass model with $\alpha=1.2$. 


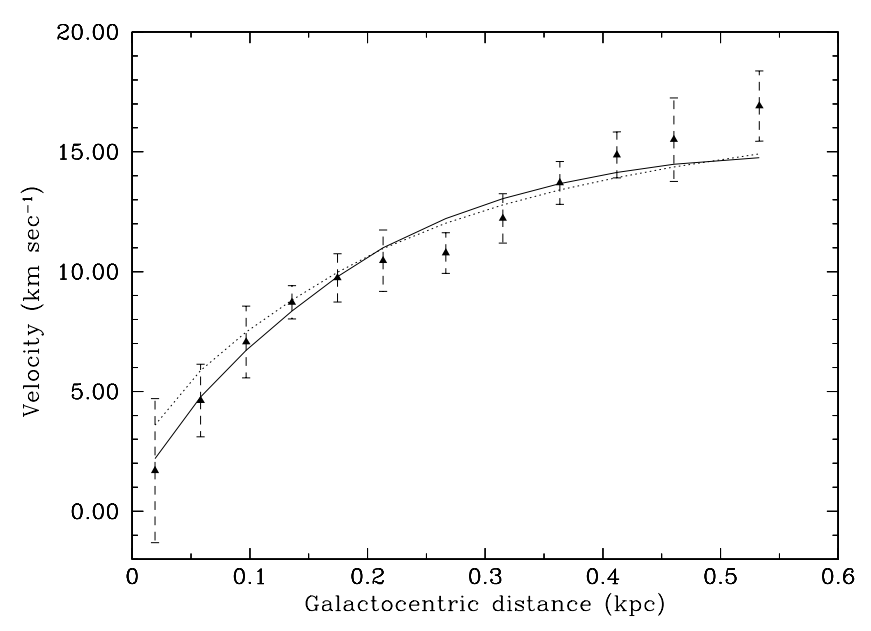

Fig. 11. The best fit MOND rotation curve (solid line) to the "asymmetric drift" corrected rotation velocities (points). Also shown in the figure is the best fit total rotation curve for a dark halo with $\alpha=1.2$ inner slope (dotted line). See the text for more details.

So far we have assumed that the discrepancy between the dynamical mass, estimated from the "asymmetric drift" corrected rotation curve, and the luminous mass in DDO210 can be explained by considering an extended dark halo around the galaxy. An alternate explanation for this discrepancy is that the dynamics become non-Newtonian in the limit of low acceleration, i.e. the MOND theory (Milgrom 1983). We have also tried fitting the rotation curve using the MOND prescription. In this fit, $\Upsilon_{B}$ and $\mathrm{a}_{0}$ (the critical acceleration parameter) were taken as free parameters. Figure 11 shows the best fit MOND rotation curve. As can be seen, the MOND rotation curve agrees well with the observed curve in the inner regions of the galaxy, while it underestimates the observed curve (by up to $2.0 \mathrm{~km} \mathrm{~s}^{-1}$ ) in the outer regions. The best fit model gave $\Upsilon_{B}$ of $0.4 \pm 0.2$ and $\mathrm{a}_{0}$ of $1.7 \pm 0.3$ (in units of $10^{-8} \mathrm{~cm} \mathrm{~s}^{-2}$ ) with reduced $\chi^{2}=0.7$. The best fit value of $\Upsilon_{B}$ agrees with the value expected from the observed colours in DDO210. Also, the best fit value of $\mathrm{a}_{0}$ is consistent with the mean value of $\mathrm{a}_{0}$ found for other, brighter galaxies (Kent 1987; see also Milgrom 1988). DDO210 is the faintest known dwarf irregular galaxy for which the MOND prescription provides a reasonably good fit to the observed kinematics.

Recall that all the above mass models have been derived by assigning an inclination of 30 degrees (obtained from the outer HI contours, as discuss in Sect. 3.1). In order to estimate the effect of using an erroneous inclination on the derived halo parameters, mass modeling was also tried with two other values of inclination viz. 60 degrees (i.e. that estimated from the optical isophotes) and 45 degrees (a value between the optical and HI inclination).

The best fit mass model for a constant density halo, using an inclination of 60 degrees, gave $\Upsilon_{B}$ of $1.4 \pm 0.2$ and $\rho_{0}=27 \pm$ $2.0 \times 10^{-3} M_{\odot} \mathrm{pc}^{-3}$ with reduced $\chi^{2}=0.3$. On the other hand, an inclination of 45 degrees gave the best fit model with $\Upsilon_{B}$ of $1.8 \pm 0.2$ and $\rho_{0}=31 \pm 2.0 \times 10^{-3} M_{\odot} \mathrm{pc}^{-3}$ with reduced $\chi^{2}=0.2$. Recall that an inclination of 30 degrees gave the best fit with $\Upsilon_{B}$ of $3.4 \pm 0.5$ and $\rho_{0}=29 \pm 5.0 \times 10^{-3} M_{\odot} \mathrm{pc}^{-3}$ with reduced $\chi^{2}=0.4$. As can be seen, the central halo density is relatively insensitive to the assumed inclination. However, the best fit $\Upsilon_{B}$ changes significantly with the assumed inclination.

For the NFW halo fit, keeping $\Upsilon_{B}$ as a free parameter in the fit gave unphysical results, hence, it was kept fixed to a value of 0.5 , as suggested by the observed colours in the galaxy. The best fit NFW model with an inclination of 60 degrees gave a reduced $\chi^{2}=4.0$, while for 45 degrees a reduced $\chi^{2}=1.6$ was obtained from the best fit. As can be seen, the NFW halo provides a poor fit to the data for higher values of inclination (for comparison, an inclination of 30 degrees gave the best fit NFW model with a reduced $\chi^{2}=0.5$ ). Hence, an NFW halo can be ruled out for DDO210, if the inclination of the galaxy is higher than 30 degrees.

\subsection{Discussion}

As discussed in Sect. 3.2, DDO210 shows signatures of twisting of the kinematical major axis as well as of kinematical lopsidedness. This may be related to the lopsidedness seen in the optical disk; the bulk of the optical emission (i.e. the "bright component" discussed in Sect. 3.1) lies in the eastern half of the HI disk. A similar pattern of kinematical lopsidedness and lopsidedness in the optical disk is also seen in other galaxies (e.g. M101, Bosma et al. 1981). To quantify the kinematical lopsidedness in the galaxy, rotation curves were derived at various spatial resolutions separately for the approaching and receding halves of the galaxy. Figure 12 shows the hybrid rotation curves for the approaching and receding sides as well as for the galaxy as a whole. The rotation curves obtained from the two lowest resolution velocity fields $\left(44^{\prime \prime} \times 37^{\prime \prime}\right.$ and $\left.29^{\prime \prime} \times 23^{\prime \prime}\right)$ matched within the errorbars with the one derived from the whole galaxy. On the other hand, for the higher resolution data, the rotation curves were found to be different (at $\sim 2.0 \mathrm{~km} \mathrm{~s}^{-1}$ level) from the rotation curve derived from the whole galaxy. While part of this difference may be due to the kinematical lopsidedness, it is also possible that part of it arises as a result of the difference in the sampling of the velocity field between the two sides of the galaxy. Because of the distorted morphology of the HI distribution, the distribution of data points available to estimate rotation velocity are different for the approaching and receding halves. This effect gets more important at the higher spatial resolutions and could also contribute to the observed differences in the rotation curves. Further, as can be seen in Fig. 12 the difference between the rotation curve derived using the whole galaxy and the rotation curves derived separately for the two halves are small compared to both the error bars on the average curve as well as the magnitude of the asymmetric drift correction. Ignoring the kinematical lopsidedness for the mass modeling is hence probably reasonable.

The derived rotation curve can also be used to put limits on the dynamical ages of the density enhancements in the HI distribution. The derived rotation curve for the galaxy (see Fig. 7) is flat from a galactocentric radii of $0.2 \mathrm{kpc}$ to $0.5 \mathrm{kpc}$. The time scales required for one rotation in the inner regions of the galaxy is $\sim 160 \mathrm{Myr}$, while the rotation period at the edge of the galaxy is $\sim 400$ Myr. Hence, this differential rotation will 


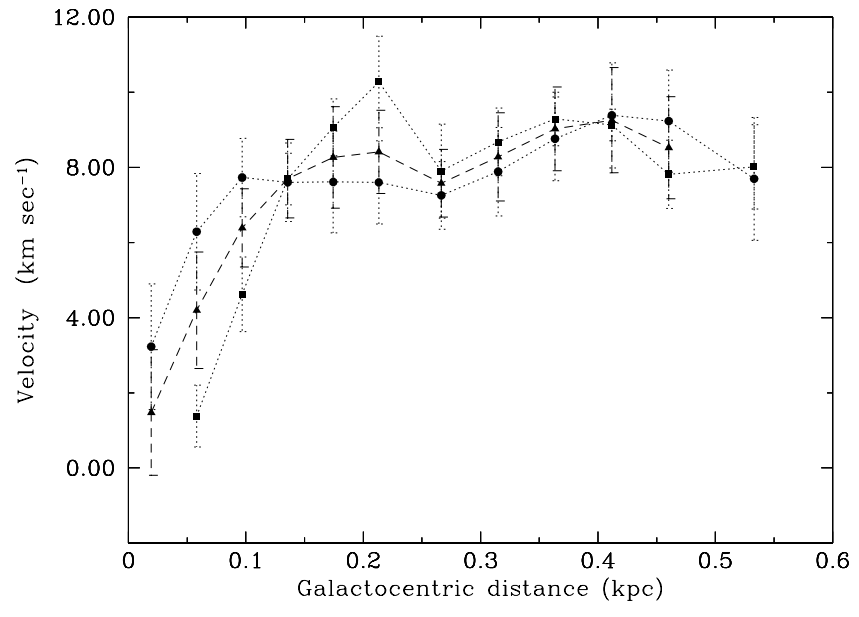

Fig. 12. Filled triangles show the rotation velocities derived from the whole galaxy while circles and squares represent the rotation velocities derived from the receding and the approaching side respectively.

wind up these density enhancements on a time scale of a few 100 Myrs. These density enhancements are hence likely to be recently developed perturbations in the HI disk. The origin of these perturbations is unknown, and it also surprising that despite their presence and relative dynamical youth there is not much evidence for star formation in the galaxy.

To conclude, we have presented deep, high velocity resolution $\left(\sim 1.6 \mathrm{~km} \mathrm{~s}^{-1}\right)$ GMRT HI $21 \mathrm{~cm}$ synthesis images for the faint $\left(M_{B} \sim-10.6\right)$ dwarf galaxy DDO210. We find that the HI distribution in the galaxy is not axi-symmetric, but shows density enhancements in the eastern and southern parts of the galaxy. The velocity field of the galaxy is however regular, and shows a systematic large scale pattern, consistent with rotational motion. The high velocity resolution $\left(\sim 1.6 \mathrm{~km} \mathrm{~s}^{-1}\right)$ and sensitivity of our observations were crucial for determining the true velocity field of the galaxy. The inclination angle for DDO210 is poorly known. From the observed velocity field, we derive a rotation curve for DDO210 by assigning an inclination derived from outer HI contours to the whole galaxy. The derived peak rotational velocity is comparable to the random motions in the HI gas. After correcting for the dynamical support provided by the random motion, we find that the rotation curve of DDO210 can be well fit with either a modified isothermal halo (with a central density $\rho_{0} \sim 29 \times 10^{-3} M_{\odot} \mathrm{pc}^{-3}$ ) or an NFW halo. We find that for a constant density halo, the central halo density is relatively insensitive to the assumed value of inclination. However, the best fit $\Upsilon_{B}$ changes significantly with the assumed inclination. On the other hand, an NFW halo can be ruled out for DDO210, if the inclination of the galaxy is higher than $\sim 30$ degrees. Mass models are also derived for a family of density profiles steeper than NFW; we find that density profiles with inner slopes steeper than $\sim 1.2$ provide a poor fit to the data.

As an alternative to the dark matter hypothesis, we have derived MOND fits to the rotation curve. We find that the rotation curve predicted by MOND provides an acceptable fit to the observed rotation curve. Further, the value of the critical acceleration $a_{0}$ of $1.7 \pm 0.3 \times 10^{-8} \mathrm{~cm} \mathrm{~s}^{-2}$ is consistent with the earlier determinations of the value of this parameter.

Acknowledgements. The observations presented in this paper would not have been possible without the many years of dedicated effort put in by the GMRT staff in order to build the telescope. The GMRT is operated by the National Centerer for Radio Astrophysics of the Tata Institute of Fundamental Research. We are grateful to Dr E. Tolstoy for providing a reduced VLT image of DDO210.

\section{References}

Begum, A., Chengalur, J. N., \& Hopp, U. 2003, New Astron., 8, 267 Bell, E. F., \& de Jong, R. S. 2001, ApJ, 550, 212

Bosma, A., Goss, M., \& Allen, R. J. 1981, A\&A, 93, 106

Bottema, R., Shostak, G. S., \& van der Kruit, P. C. 1986, A\&A, 167, 34

Casertano, S. 1983, MNRAS, 203, 735

Côté, S., Carignan, C., \& Freeman, K. C. 2000, ApJ, 120, 3027

de Grijs, R., \& Peletier, R. F. 1997, A\&A, 320, L21

Fisher, J. R., \& Tully, R. B. 1975, A\&A, 44, 151

Fisher, J. R., \& Tully, R. B. 1979, AJ, 84, 62

Greggio, L., Marconi, G., Tosi, M., \& Focardi, P. 1993, AJ, 105, 894

Huchtmeier, W. K., \& Richter, O. G. 1986, A\&AS, 63, 323

Karachentsev, I. D., Sharina, M. E., Makarov, D. I., et al. 2002, A\&A, 389, 812.

Kent, S. M. 1987, AJ, 93, 816

Kravtsov, A. V., Klypin, A. A., Bullock, J. S., \& Primack, J. R. 1998, ApJ, 502, 48

Lake, G., Schommer, R. A., \& van Gorkom, J. H. 1990, AJ, 99, 547

Lee, M. G., Aparicio, A., Tikonov, N., Byun, Y., \& Kim, E. 1999, AJ, 118,853

Lo, K. Y., Sargent, W. L. W., \& Young, K. 1993, ApJ, 106, 507

Marchesini, D., D’Onghia, E., Chincarini, G., et al. 2002, ApJ, 575, 801

Mateo, M. 1998, ARA\&A, 36, 506

Moore, B., Quinn, T., Governato, F., Stadel, J., \& Lake, G. 1999, MNRAS, 310, 1147

Milgrom, M. 1983, ApJ, 260, 365

Milgrom, M. 1988, ApJ, 333, 689

Navarro, J. F., Frenk, C. S., \& White, S. D. M. 1996, ApJ, 462, 563-575

Skillman, E. D., Neutral Hydrogen in Dwarf Galaxies, 1996, PASP Conf. Ser., 106, 208

Skillman, E. D., Terlevich, R., Teuben, P. J., \& van Woerden, H. 1988, A\&A, 198, 33

Swarup, G., Ananthakrishnan, S., Kapahi, V. K., et al. 1991, Curr. Sci., 60,95

Swaters, R. A., Schoenmakers, R. H. M., Sancisi, R., \& van Albada, T. S. 1999, MNRAS, 304, 330

Taylor, C. L., Kobulnicky, H. A., \& Skillman, E. D. 1998, AJ, 116, 2746

Tolstoy, E., Gallagher, J., Greggio, L., et al. 2000, ESO Messenger, 99,16

van Zee, L., Haynes, M. P., \& Salzer, J. J. 1997, AJ, 114, 2479

van Zee, L. 2000, AJ, 119, 2757

van den Bergh, S. 1959, Pub. David Dunlap Obs., 2, 147

van den Bosch, F. C., \& Swaters, R. A. 2001, MNRAS, 325, 1017

van der Kruit, P. C., \& Searle, L. 1981, A\&A, 95, 105

Weldrake, D. T. F., de Blok, W. J. G., \& Walter, F. 2003, MNRAS, 340,12

Young, L. M., van Zee, L., Lo, K. Y., Dohm-Palmer, R. C., \& Beierle, M. E. 2003 [astro-ph/0304266] 